\title{
DEGLUTIÇÃO APÓS QUIMIOTERAPIA E RADIOTERAPIA SIMULTÂNEA PARA CARCINOMAS DE LARINGE E HIPOFARINGE
}

\author{
Andréa Bizarria Cintra, luciana Passuello do Vale, Olavo Feher, Inês Nobuko Nishimoto, \\ Luiz Paulo Kowalski, Elisabete Carrara de Angelis* \\ Trabalho realizado no departamento de Cirurgia de Cabeça e Pescoço e Otorrinolaringologia \\ do Centro de Tratamento e Pesquisa do Hospital do Câncer AC Camargo, São Paulo, SP
}

RESUMO - OBJetivo. Avaliar a deglutição orofaríngea de pacientes submetidos a protocolo de preservação de laringe no Hospital do Câncer AC Camargo.

Métodos. De 43 pacientes com carcinoma epidermóide de laringe e hipofaringe tratados com quimioterapia semanal (paclitaxel $30 \mathrm{mg} / \mathrm{m}^{2}$ e cisplatina $20 \mathrm{mg} / \mathrm{m}^{2}$ ) concomitante à radioterapia externa fracionada (180 cGy/dia, dose total 7040 cGy), foram avaliados 31 pacientes. A avaliação consistiu de videofluoroscopia. Os resultados foram classificados de acordo com alterações de motilidade orofaríngea, estases, aspiração e severidade da disfagia.

Resultados. Alterações de fase preparatória e oral: estase em cavidade oral (58\%) e aumento do tempo de trânsito oral $(32,3 \%)$; fase faríngea: estase em valécula $(83,9 \%)$, redução da elevação laríngea $(74,2 \%)$, atraso no início da deglutição faríngea $(51,6 \%)$ e estase em hipofaringe (45,2\%). A deglutição funcional foi observada em $35,5 \%$ dos pacientes, $54,8 \%$ tinham disfagias discretas ou moderadas e $9,6 \%$ disfagias severas.

Conclusão. A associação de quimioterapia e radioterapia para preservação laríngea resulta em alterações da deglutição, na maior parte discretas a moderadas. Alguns pacientes, entretanto, evoluem com disfagia severa, em que a alimentação por via oral não é possível.

UnITERMOS: Deglutição/fisiologia. Preservação de órgãos. Câncer de cabeça e pescoço. Quimioterapia. Radioterapia.

\section{INTRODUÇÃO}

A laringe tem como função primária a respiração, mas desenvolveu a fonação como função secundária, além de participar no mecanismo de proteção das vias aéreas inferiores durante o processo de deglutição. Este processo pode ser dividido em quatro fases: preparatória - fase voluntária em que é formado o bolo alimentar com a trituração dos alimentos e sua mistura à saliva; oral - fase voluntária em que o bolo alimentar é posicionado e projetado para trás através de movimentos ondulatórios da língua; faríngea - fase involuntária que se inicia com o disparo do reflexo da deglutição, seguida da elevação e anteriorização da laringe e da abertura do esfíncter esofágico superior para a passagem do bolo; esofágica - fase involuntária em que o bolo é levado por movimentos peristálticos até o estômago.

Segundo Crespo', o câncer da laringe causa grande impacto na função de deglutição e

\footnotetext{
*Correspondência:

Departamento de Fonoaudiologia

Rua Professor Antônio Prudente, 211

CEP: 01509-900 - São Paulo - SP - fax: 3272-5124 e-mail: eangeli@attglobal.net
}

também na qualidade de vida. $\mathrm{O}$ tratamento tradicional para o câncer avançado de laringe é a laringectomia total ${ }^{2}$. Estudos recentes mostram a viabilidade da preservação do órgão através de tratamentos combinados de radioterapia e quimioterapia, sem comprometimento dos resultados em termos de tempo de sobrevida ${ }^{3,4,5}$. No entanto, a laringe preservada nem sempre é funcional.

Em pacientes submetidos à radioterapia exclusiva, são observadas fibroses da musculatura e tecidos moles da faringe e laringe, além de xerostomia. Essas alterações podem acarretar modificações na fisiologia da deglutição orofaríngea, com aumento do tempo de trânsito faríngeo do alimento, elevação laríngea reduzida e penetrações ou aspirações laríngeas, entre outros ${ }^{6,7,8}$.

A partir da década de 1990, começaram a surgir os estudos que avaliavam a deglutição dos pacientes submetidos a protocolos de preservação. Esses estudos utilizaram como metodologia questionários e/ou avaliação videofluoroscópica. Os autores são unânimes ao associar as alterações da motilidade oral e faríngea encontradas como decorrentes do tratamento radioterápico, embora esses aspectos piorem quando se associam à radio- terapia e à quimioterapia ${ }^{6,9-14}$. Descrever a fisiologia da deglutição nesses pacientes é importante para o conhecimento das seqüelas e melhor planejamento da conduta terapêutica. Além disso, é importante também destacar a necessidade de avaliação dos custos de cada modalidade de tratamento, a fim de obter dados mais reais para uma posterior comparação com as modalidades tradicionais de tratamento (cirurgia).

Este estudo tem por objetivo avaliar a deglutição dos pacientes submetidos ao protocolo de preservação de órgãos por tumores avançados de laringe e/ou hipofaringe do Centro de Tratamento e Pesquisa do Hospital do Câncer AC Camargo.

\section{Métodos}

Entre os meses de outubro de 1999 e outubro de 200I, foram admitidos no Centro de Tratamento e Pesquisa do Hospital do Câncer AC Camargo 43 pacientes elegíveis para o protocolo de preservação de laringe, portadores de carcinomas epidermóides avançados de laringe e hipofaringe.

O tratamento se constituiu de doses semanais de paclitaxel $\left(30 \mathrm{mg} / \mathrm{m}^{2}\right)$ e cisplatina (20 $\mathrm{mg} / \mathrm{m}^{2}$ ) concomitante a doses de radioterapia 


\begin{tabular}{|c|c|c|c|c|c|}
\hline \multicolumn{6}{|c|}{ Tabela I - Caracterização dos pacientes } \\
\hline $\begin{array}{c}\text { Paciente }\left(\mathrm{n}^{\circ}{ }^{\circ}\right) \\
\text { idade(anos)/sexo }\end{array}$ & TNM & Local do tumor & $\begin{array}{c}\text { Intervalo RxT/VF } \\
\text { (dias) }\end{array}$ & $\begin{array}{l}\text { Dias de } \\
\text { traqueostomia }\end{array}$ & $\begin{array}{c}\text { Dias de } \\
\text { sonda enteral }\end{array}$ \\
\hline $01 / 66 / \mathrm{M}$ & $\overline{\text { T3NOMO }}$ & Transglótico & 140 & & \\
\hline 02/59M M & T3NOMO & Glótico & 112 & & 122 \\
\hline 03/56/M & T3NOMO & Transglótico & 84 & 395 & \\
\hline 04/65/M & T3NOMO & Supra-glótico & 140 & & \\
\hline 05/73/M & T4NIMO & Seio piriforme & 84 & 6 & 122 \\
\hline $06 / 52 / M$ & T4N2aMO & Seio piriforme & 56 & & 91 \\
\hline $07 / 69 M$ & T3NOMO & Glótico & 196 & & \\
\hline 08/54/M & T3NOMO & Transglótico & 280 & 210 & \\
\hline $09 / 61 / M$ & T4N2bMO & Transglótico & 308 & & \\
\hline $10 / 72 / \mathrm{F}$ & TANOMO & Transglótico & 84 & 212 & 213 \\
\hline $1 / / 56 / M$ & T3NIMO & Transglótico & 224 & 152 & \\
\hline $12 / 39 / M$ & T4NOMO & Transglótico & 280 & & \\
\hline $13 / / 52 M$ & T3N2aMO & Supra-glótico & 168 & & 5 \\
\hline $14 / 57 / M$ & T3NIMO & Supra-glótico & 448 & & \\
\hline$|5 / 6| / M$ & T3NIMO & Transglótico & 28 & & 30 \\
\hline$|6 / 4| / M$ & T4NOMO & Transglótico & 168 & 91 & 61 \\
\hline $17 / 58 / M$ & T3NOMO & Transglótico & 84 & & \\
\hline $18 / 43 / \mathrm{M}$ & T3N2aMO & Seio piriforme & । & & \\
\hline 19/57/M & T3NOMO & Glótico & 84 & & 91 \\
\hline 20/63/M & T4NZaMO & Supra-glótico & 112 & & 122 \\
\hline $21 / 59 / M$ & T3NOMO & Seio piriforme & 112 & & \\
\hline 22/69/M & T3NOMO & Glótico & 196 & 60 & \\
\hline 23/47/M & T4NOMO & Glótico & 56 & 121 & \\
\hline $24 / 57 / M$ & T4NOMO & Transgglótico & 13 & & 213 \\
\hline 25/66/M & T3N2aMO & Seio piriforme & 196 & & 122 \\
\hline $26 / 64 / F$ & T3NOMO & Glótico & 252 & 6 & 61 \\
\hline $27 \pi 2 / F$ & T3NOMO & Supra-glótico & 6 & & 213 \\
\hline 28/6/M & T4NOMO & Transglótico & 84 & & \\
\hline 29/62/M & T3N2bMO & Transglótico & 140 & & \\
\hline $30 / 67 / M$ & T3NOMO & Transglótico & 84 & & \\
\hline $31 / 44 / M$ & T4NIMO & Seio piriforme & 112 & & 61 \\
\hline
\end{tabular}

fracionada (I80 cGy/fração/dia) numa dose total de 7040 cGy.

Desses 43 pacientes, 31 aceitaram voluntariamente realizar a avaliação da deglutição. Inicialmente foi realizado um levantamento de prontuários no Serviço de Arquivo Médico e Estatística (SAME), onde foram coletados dados de identificação (sexo, idade, RGH, entre outros), diagnóstico médico, diagnóstico laringológico, queixas quanto à deglutição e à voz e tratamentos realizados. As características principais dos pacientes são descritas na Tabela I. A faixa etária variou de 39 a 73 anos (média de 59 anos), sendo 27 homens e 4 mulheres. Todos foram submetidos à avaliação videofluoroscópica da deglutição entre I dia e I 4 meses após a conclusão do tratamento radioquimioterápico. Nenhum paciente apresentava no momento do exame história de doenças neurológicas, sinais clínicos de recorrência tumoral no sítio primário, disfunções gastroentereológicas, e também não haviam realizado fonoterapia para deglutição ou voz.

Quanto ao estadiamento de acordo com a classificação TNM da UICC (2002), a amostra do estudo constou de 13 pacientes T3N0, três T3NI, dois T3N2a, dois T3N2b, seis T4NO, dois T4NI, dois T4N2a e um T4N2b. Não houve casos com metástase a distância no momento da admissão.

Durante o período de tratamento, nove pacientes necessitaram realizar traqueostomia. Seu tempo de uso variou de 6 dias a 12 meses (média de 40 dias). A gastrostomia foi utilizada em um paciente e a sonda enteral foi necessária para suporte nutricional em 13 , e seu tempo de uso variou entre 5 dias e 17 meses (média de 50 dias).
A avaliação da deglutição foi realizada com equipamento radiológico do modelo I600E, GE Medical Systems, Milwaukee, Wis, por uma fonoaudiológa e um médico radiologista. Para a análise da deglutição, foram consideradas as primeiras videofluoroscopias realizadas após a conclusão do protocolo de preservação de laringe. Todas as avaliações seguiram o mesmo protocolo de exame utilizado pela instituição: o foco da imagem fluoroscópica foi definido anteriormente pelos lábios, superiormente pelo palato duro, posteriormente pela parede posterior de faringe, inferiormente pela bifurcação entre o esfíncter esofágico superior e a abertura da via aérea aproximadamente no nível da sétima vértebra cervical. Os pacientes foram posicionados em pé, orientados a deglutir o material oferecido após comando 


\begin{tabular}{|c|c|c|}
\hline \multicolumn{3}{|c|}{ Quadro I - Escala de penetração e aspiração (Rosenbek et al., 1996) } \\
\hline Categoria & Pontuação & Descrição \\
\hline \multirow[t]{5}{*}{$\overline{\text { Penetração }}$} & 1 & Contraste não entra em via aérea. \\
\hline & 2 & Contraste entra até acima das ppwv, sem resíduo. \\
\hline & 3 & Contraste permanece acima das ppw, resíduo visível. \\
\hline & 4 & Contraste atinge ppvv, sem resíduo. \\
\hline & 5 & Contraste atinge ppw, resíduo visível. \\
\hline \multirow[t]{3}{*}{ Aspiração } & 6 & $\begin{array}{l}\text { Contraste passa o nível glótico, mas não há resíduo } \\
\text { no nível subglótico. }\end{array}$ \\
\hline & 7 & $\begin{array}{l}\text { Contraste passa o nível glótico com resíduo no nível subglótico } \\
\text { apesar do pc responder. }\end{array}$ \\
\hline & 8 & $\begin{array}{l}\text { Contraste passa a glote com resíduo na subglote, mas o pc } \\
\text { não responde. }\end{array}$ \\
\hline
\end{tabular}

ppw: pregas vocais; pc: paciente

\section{Quadro 2 - Escala de severidade das disfagias (O'Neil et al., 1999)}

\section{O.: DIETANORMAL}

nível 7 Normal em todas as situações. O paciente não necessita de estratégías ou tempo extra.

nível 6 Deglutição funcional. O pc pode ter discreto atraso oral ou faríngeo, estase ou vestígio cobrindo a epiglote, mas espontaneamente o limpa. Pode necessitar de tempo extra para as refeições, mas não há penetração e/ou aspiração em todas as consistências.

\section{0.: DIETAMODIFICADAE/OU INDEPENDÊNCIA}

nível 5 Disfagia discreta. O pc pode necessitar de supervisão a distancia, com restrição de uma consistência. Pode demonstrar aspiração somente a líquido, mas com forte reflexo de tosse; penetração acima das ppw; com uma ou mais consistências, ou sobre as ppw; com uma consistência, mas com clareamento espontâneo; redução da mastigaçãa e/ou estase oral que limpa espontaneamente.

Nível 4 Disfagia discreta/moderada. O pc necessita de total supervisão (estratégias) com restrição a duas ou mais consistências. Pode ter estase moderada em faringe ou CO que demandem orientação para a limpeza; aspiração com uma consistência com reflexo de tosse fraco ou ausente; penetração em ppw. Com tosse com duas consistências; penetração em ppw. Em todas as consistências sem tosse.

Nível 3 Disfagia moderada. O pc necessita de total supervisão (estratégias) com restrição a duas ou mais consistências. Pode ter estase moderada em faringe e/ou CO com clareamento sob orientação; penetração em ppw. Sem tosse com duas consistências com reflexo de tosse fraco ou ausente; aspiração com uma consistência sem tosse na penetração.

\section{0. SUSPENSA: NECESSIDADE DE NUTRIÇÄO ENTERAL}

nível 2 Disfagia moderada/severa. O pc necessita de supervisão contínua (estratégias) com VO parcial (treino). Pode ter estase severa na faringe, sendo incapaz de clarear ou necessitar de vários comandos; estase severa ou perda prematura do bolo na fase oral, sendo incapaz de clarear ou necessitar de vários comandos; penetração em ppw. Com uma ou mais consistências, sem tosse; aspiração com duas ou mais consistências sem reflexo de tosse ou tosse voluntária fraca.

Nível I Disfagia severa. O pc é incapaz de tolerar dieta VO com segurança. Pode ter estase severa na faringe, estase ou perda prematura do bolo em fase oral, incapaz de clarear; aspiração silente com duas ou mais consistências e tosse voluntária não funcional; incapaz de conseguir executar a deglutição.

ppw: pregas vocais; pc: paciente

verbal. Foram realizadas deglutições nas visões lateral e ântero-posterior e as imagens gravadas em fita de VHS. O material utilizado constituiu-se de bário líquido (5 e $20 \mathrm{ml}$ ) misturado com água numa proporção de $1: 1$ para a consistência líquida; para a consistência líquido-pastosa ( $5 \mathrm{ml}$ ) utilizou-se o bário líquido; para a pastosa $(5 \mathrm{ml})$ utilizou-se bário pasta; e para sólida, um pedaço de bolacha waffle misturada ao bário pasta.

A análise dos exames foi realizada por três fonoaudiólogas com experiência na área, que assistiram no mesmo momento às gravações dos exames dos pacientes e juntas classificaram as alterações observadas nas fases preparatória-oral, oral e faríngea de acordo com os achados propostos por Robbins, Logemann e Kirshner (1985).

Para a graduação das penetrações e aspirações laríngeas utilizou-se a escala de penetração e aspiração proposta por Rosenbek et al. (1996) (Quadro I). Para a análise do grau das disfagias foi utilizada a escala proposta por O'Neil et al. (1999) (Quadro 2).
Estatísticas descritivas de freqüências absolutas e relativas foram utilizadas para descrever as variáveis categóricas. As variáveis numéricas ou contínuas foram representadas pelas medidas de tendência central e de variabilidade. As associações entre avaliações com relação às variáveis categóricas foram verificadas através do teste de frequiências exato de Fisher e a avaliação das disfagias segundo o tempo, em meses, pós-tratamento, pelo teste não paramétrico de Kruskal-Wallis, considerando-se nível de significância de 5\% ( $p<0.05)$. 


\section{Tabela 2 - Distribuição das freqüências quanto às alterações observadas nas fases preparatória-oral, oral e faríngea}

\begin{tabular}{|c|c|c|c|}
\hline \multirow[t]{2}{*}{ Variável } & \multirow[t]{2}{*}{ Categoria } & \multicolumn{2}{|c|}{ Freqüência(\%) } \\
\hline & & Sim & Não \\
\hline Alterações de fase preparatória-oral & $\begin{array}{c}\text { ECO } \\
\text { PPB } \\
\text { AFB } \\
10\end{array}$ & $\begin{array}{l}9(29 \%) \\
5(16 \%) \\
4(13 \%) \\
1(3 \%)\end{array}$ & $\begin{array}{l}22(71 \%) \\
26(84 \%) \\
27(87 \%) \\
30(97 \%)\end{array}$ \\
\hline Alterações de fase oral & $\begin{array}{l}\text { ATTO } \\
\text { RMAPL } \\
\text { RCLP } \\
\text { ECO }\end{array}$ & $\begin{array}{c}10(32 \%) \\
1(3 \%) \\
3(9,7 \%) \\
18(58)\end{array}$ & $\begin{array}{c}21(68 \%) \\
30(97 \%) \\
28(90,3 \%) \\
13(42 \%)\end{array}$ \\
\hline Alterações de fase faríngea & $\begin{array}{c}\text { ATTF } \\
\text { AFL } \\
\text { AFVL } \\
\text { ERP } \\
\text { EA } \\
\text { REL } \\
\text { EV } \\
\text { EPPF } \\
\text { EBL } \\
\text { AIDF }\end{array}$ & $\begin{array}{c}3(9,7 \%) \\
3(9,7 \%) \\
7(22,6 \%) \\
5(16 \%) \\
10(32 \%) \\
23(74 \%) \\
26(84 \%) \\
14(45 \%) \\
13(42 \%) \\
16(52 \%)\end{array}$ & $\begin{array}{c}28(90,3 \%) \\
28(97,3 \%) \\
24(77,4 \%) \\
26(84 \%) \\
21(68 \%) \\
8(26 \%) \\
5(16 \%) \\
17(55 \%) \\
18(58 \%) \\
15(48 \%)\end{array}$ \\
\hline
\end{tabular}

No total da amostra: 31 pacientes

- ECO - estase em cavidade oral; PPB - perda prematura do bolo; AFB - alteração na formação do bolo; 10 - incontinência oral.

- ATTO - aumento do tempo de trânsito oral; RMAPL - redução do movimento ântero-posterior de língua; RCLP - redução do contato de língua x palato; ECO - estase em cavidade oral.

- ATTF - aumento do tempo de trânsito faríngeo: AFL - alteração de fechamento laríngeo; AFVL - alteração de fechamento de vestíbulo laríngeo; ERP - estase em recessos piriformes; EA - estase em aritenóides; REL - redução de elevação laríngea; EV - estase em valécula; EPPF - estase em parede posterior de faringe; EBL - estase em base de língua; AIDF - atraso no início da deglutição faríngea.

Figura I - Presença de penetração e/ou aspiração laríngea (Rosenbek et al., 1996) em pacientes submetidos ao protocolo de preservação de órgãos
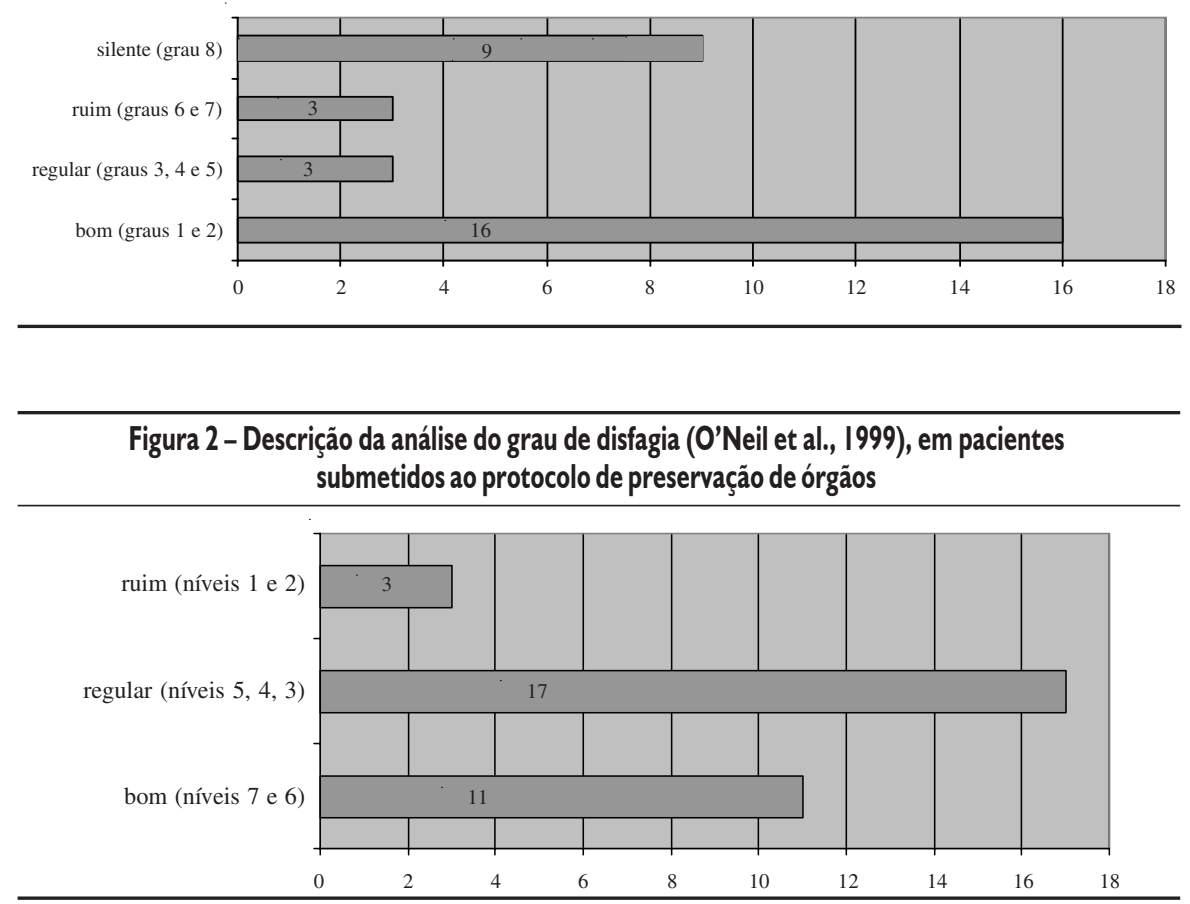

\section{Resultados}

Todos os pacientes selecionados para esse estudo apresentaram resposta completa ao tratamento, sem presença de tumor detectável à avaliação na época em que foi realizada a videofluoroscopia.

A Tabela 2 apresenta as alterações observadas na fase preparatória oral, com presença de estases em nove casos (29\%), seguida de perda prematura do bolo em cinco casos $(16,1 \%)$. Na fase oral, as estases mantiveram-se como a alteração mais observada ( 8 casos, 58\%).

É na fase faríngea que foram encontradas as maiores alterações: a) estase em valécula (26 casos, 83,9\%); b) redução da elevação laríngea em 23 casos (74,2\%); c) atraso no início da deglutição faríngea em 16 casos (51,6\%). As estases em parede posterior de faringe, em base de língua e em aritenóides foram observadas em I4 (45,2\%), 13 $(41,9 \%)$ e $10(32,3 \%)$ casos, respectivamente. As menores ocorrências observadas foram penetração nasal e redução do contato de língua com a faringe (um caso cada, 3,2\%) (Tabela 2).

A Figura I apresenta a distribuição dos pacientes quanto às penetrações e/ou aspirações laríngeas de acordo com a escala de Rosenbek et al. (1996). Em 16 casos (51\%) a classificação obtida foi "boa", com o restante se dividindo nas outras categorias. A avaliação final das disfagias é apresentada na Figura 2, baseada na escala de O'Neil et al. (1999). No grupo estudado, I I pacientes $(35,5 \%)$ concentraram-se na categoria "bom", sendo que estes obtiveram na escala avaliação nível 6, ou seja, uma deglutição funcional. Esses pacientes tendem a ter tempo inferior de intervalo entre o final do tratamento e a avaliação videofluoroscópica, mas a diferença não foi significativa (Tabela 5).

A deglutição de 17 pacientes da amostra $(54,8 \%)$ foi classificada na categoria "regular", sendo que, desses, sete $(22,6 \%)$ obtiveram nível 5 (disfagia discreta), e 10 (32,3\%) obtiveram nível 4 (disfagia discreta/moderada). Apenas três pacientes (9,7\%) tiveram sua deglutição classificada como "ruim", necessitando suspender a via oral de alimentação. Do grupo de estudo, nove pacientes apresentaram recidiva local diagnosticada posteriormente. Desses pacientes, oito (88,9\%) apresentaram avaliação de penetração/aspiração laríngea considerada boa. Não houve 


\begin{tabular}{|c|c|c|c|c|c|}
\hline $\begin{array}{l}\text { Avaliação final } \\
\text { das disfagias }\end{array}$ & $\mathbf{N}$ & Variação & $\begin{array}{l}\text { Medidas } \\
\text { Mediana }\end{array}$ & Média (dp) & P* \\
\hline $\begin{array}{c}\text { Bom } \\
\text { Regular } \\
\text { Ruim }\end{array}$ & $\begin{array}{l}11 \\
17 \\
3\end{array}$ & $\begin{array}{c}\text { I dia-10 } \\
6 \text { dias - 15,4 } \\
2,9-7,4\end{array}$ & $\begin{array}{l}2,9 \\
5,1 \\
4,4\end{array}$ & $\begin{array}{l}3,6(2,9) \\
5,8(4,0) \\
4,9(2,3)\end{array}$ & 0,2128 \\
\hline
\end{tabular}

*Teste de Kruskal-Wallis

\begin{tabular}{|c|c|c|c|c|}
\hline \multirow[t]{2}{*}{ Variável } & \multirow[t]{2}{*}{ Categoria } & \multicolumn{2}{|c|}{$\begin{array}{c}\text { Avaliação das } \\
\text { penetraçōes/aspiraçōes laríngeas }\end{array}$} & \multirow[t]{2}{*}{$\mathbf{P}$} \\
\hline & & Níveis I a 5 & Níveis 6 a 8 & \\
\hline Estadiamento $\mathrm{T}$ & $\begin{array}{l}\text { T3 } \\
\text { T4 }\end{array}$ & $\begin{array}{l}13(68 \%) \\
6(32 \%)\end{array}$ & $\begin{array}{l}7(58 \%) \\
5(42 \%)\end{array}$ & 0,705 \\
\hline Local do tumor & $\begin{array}{l}\text { Laringe } \\
\text { Hipofaringe }\end{array}$ & $\begin{array}{l}17(89 \%) \\
2(11 \%)\end{array}$ & $\begin{array}{l}8(67 \%) \\
4(33 \%)\end{array}$ & 0,174 \\
\hline Recidiva local & $\begin{array}{l}\text { Não } \\
\text { Sim }\end{array}$ & $\begin{array}{l}11(58 \%) \\
8(42 \%)\end{array}$ & $\begin{array}{l}\text { II (92\%) } \\
\text { | (8\%) }\end{array}$ & 0,101 \\
\hline Óbito & $\begin{array}{l}\text { Não } \\
\text { Sim }\end{array}$ & $\begin{array}{l}15(79 \%) \\
4(21 \%)\end{array}$ & $\begin{array}{l}8(67 \%) \\
4(33 \%)\end{array}$ & 0,676 \\
\hline
\end{tabular}

*Teste exato de Fisher

\begin{tabular}{|c|c|c|c|c|}
\hline \multirow[t]{2}{*}{ Variável } & \multirow[t]{2}{*}{ Categoria } & \multicolumn{2}{|c|}{ Avaliação das disfagias } & \multirow[t]{2}{*}{ P* } \\
\hline & & Nível bom & Nível regular/ruim & \\
\hline Estadiamento T & $\begin{array}{l}\text { T3 } \\
\text { T4 }\end{array}$ & $\begin{array}{l}7(64 \%) \\
4(36 \%)\end{array}$ & $\begin{array}{c}13(65 \%) \\
7(35 \%)\end{array}$ & 0,999 \\
\hline Local do tumor & $\begin{array}{l}\text { Laringe } \\
\text { Hipofaringe }\end{array}$ & $\begin{array}{l}9(82 \%) \\
2(18 \%)\end{array}$ & $\begin{array}{l}16(80 \%) \\
4(20 \%)\end{array}$ & 0,999 \\
\hline Recidiva local & $\begin{array}{l}\text { Não } \\
\text { Sim }\end{array}$ & $\begin{array}{l}5(46 \%) \\
6(54 \%)\end{array}$ & $\begin{array}{l}17(85 \%) \\
3(15 \%)\end{array}$ & 0,038 \\
\hline Óbito & $\begin{array}{l}\text { Não } \\
\text { Sim }\end{array}$ & $\begin{array}{l}9(82 \%) \\
2(18 \%)\end{array}$ & $\begin{array}{l}14(70 \%) \\
6(30 \%)\end{array}$ & 0,676 \\
\hline
\end{tabular}

*Teste exato de Fisher

diferença significativa com os casos que permaneceram sem recorrência $(p=0,10 \mid)$ (Tabela 3).

Entre os pacientes que não apresentaram recidiva local, 17 (77,3\%) tiveram avaliação final da disfagia classificada como boa ou regular; já entre os pacientes que apresentaram recidiva local, seis $(66,7 \%)$ tiveram a avaliação da disfagia classificada como ruim (Tabela 4).

\section{Discussão}

Os estudos realizados com protocolos de preservação de órgãos vêm demonstrando taxas de sobrevida semelhantes ao tratamento
A casuística do estudo foi composta por 3 | pacientes com tumores avançados de laringe ou hipofaringe, sendo a maior parte deles tumores transglóticos (14 casos, 45,16\%). Todos os pacientes, independentemente da queixa, foram avaliados, diferentemente dos estudos anteriores que avaliaram apenas pacientes com disfagia grave 3,4,5,6,9-13. O grupo de estudo realizou a avaliação entre um dia e 14 meses após o término do tratamento (média de quatro meses). Durante o período do tratamento, nove (29\%) pacientes da amostra necessitaram de uso da traqueostomia, cujo período variou entre 6 dias e 12 meses (média de 40 dias). A gastrostomia foi utilizada em um paciente e o uso de sonda nasoenteral para alimentação foi necessário em 13 (45,2\%), e seu período de uso variou entre cinco dias a sete meses (média de 50 dias). No momento da avaliação, 10 pacientes (32,6\%) ainda estavam utilizando sonda nasoenteral, mas nenhum utilizava-se de gastrostomia.

Em nosso estudo, apesar dos tumores se localizarem em regiões mais inferiores (laringe e hipofaringe), foram observadas alterações da deglutição em todas as fases do processo, variando quanto à freqüência de ocorrências. $\mathrm{Na}$ fase preparatória-oral, a estase em cavidade oral foi a alteração de maior ocorrência, com nove casos (29\%). Essa alteração se justifica pela redução na sensibilidade orofaríngea e/ou pela xerostomia advindas da radioterapia. De acordo com Lazarus ${ }^{9}$, isso ocorre pois a radioterapia atinge também regiões adjacentes de laringe e base de língua, alterando dessa forma os tecidos normais.

$\mathrm{Na}$ fase oral, as estases em cavidade oral foram as alterações observadas com maior freqüência: em 18 casos (58\%). Também foram observados 10 casos (32,3\%) em que ocorreu o aumento no tempo de trânsito oral. Um dos efeitos da radioterapia mais observados na fase crônica é a fibrose da musculatura que leva à redução da mobilidade e justifica o aumento do tempo da deglutição, também relatado na literatura ${ }^{6,7,9,13,19}$. Estes achados também podem estar associados à xerostomia, redução de sensibilidade orofaríngea e alteração da formação do bolo na fase preparatória oral.

Como esperado, na fase faríngea foram encontradas as maiores freqüências de alterações (Tabela 3) devido ao fato de o tratamento 
se concentrar nessa região. Essas alterações podem ser agrupadas e justificadas como decorrentes de dois fatores, a saber: alterações decorrentes de redução na sensibilidade (estases, principalmente em valécula e parede posterior de faringe, e atraso no início da deglutição faríngea) e alterações decorrentes de redução na mobilidade (estases, alteração de fechamento de vestíbulo, redução da elevação laríngea). Em nosso estudo, foi observada a estase em valécula, parede posterior de faringe, e em recessos piriformes em 26 casos (83,9\%), I 4 casos ( $45,2 \%$ ) e 5 casos ( $16,1 \%)$, respectivamente. Esses dados são semelhantes aos relatados nos estudos de Lazarus ${ }^{8}$, com nove pacientes, que comparados com um grupo controle apresentaram alterações de fase oral e faríngea na deglutição. A elevação laríngea reduzida foi a alteração de mobilidade com maior ocorrência (23 casos 74,2\%), alteração também vista nos achados de Kotz com 15 pacientes.

$\mathrm{Na}$ classificação das penetrações e aspirações laríngeas, os pacientes foram agrupados em quatro grupos de acordo com a escala de Rosenbek et al. (1996), sendo bom (graus I e 2), regular (graus 3, 4 e 5), ruim (graus 6 e 7), e silente (grau 8). Alguns estudos anteriores citam grande índice de penetrações e/ou aspirações laríngeas ${ }^{6,9,13,20}$. De acordo com a classificação utilizada, podemos observar que os pacientes concentram-se no grupo bom, ou seja, não têm ou quando apresentam penetração laríngea, limpam espontaneamente as estases (Figura I). Nesses pacientes, a redução da sensibilidade parece não exercer grande influência, ou talvez o aumento do tempo de trânsito faça com que o indivíduo se "prepare" melhor e evite a penetração/aspiração laríngea. Três pacientes $(9,6 \%)$ apresentaram nível de penetração/aspiração regular, ou seja, apresentavam a entrada do alimento até a altura de pregas vocais, com ou sem resíduos. Três pacientes $(9,6 \%)$ apresentaram deglutição classificada em nível ruim, com aspirações, com ou sem resíduos, e sintomáticas. Nove pacientes (29\%) apresentaram aspirações silentes, indicando alteração da sensibilidade laríngea, decorrente da radioterapia ou da dessensibilização que ocorre em função da aspiração crônica.

Para classificar as disfagias, agrupamos os pacientes em três grupos seguindo a escala proposta por O'Neil et al., em 1999: bom (via oral normal); regular (via oral modificada); ruim (via oral suspensa). Observamos I I pacientes $(35,5 \%)$ com nível bom de deglutição (nível 6), ou seja, embora possam apresentar estases e discreto aumento do tempo de trânsito oral ou faríngeo, normalmente realizam a limpeza espontaneamente e conseguem alimentar-se via oral em todas as consistências. No grupo, não foram observadas diferenças significativas quanto ao tempo de intervalo entre radioterapia e videofluoroscopia, apesar do grupo "bom" tender a ter tempo inferior. Cerca de metade dos pacientes ( 17 casos, $54,8 \%$ ) foi julgada com nível regular de deglutiçãa (sete pacientes no nível 5 , sete nível 4 e três nível 3), com maior grau de estases, dificuldade no clareamento orofaringolaríngeo e presença de penetrações/aspirações, já restringindo a alimentação de algumas consistências, mas ainda mantendo alimentação via oral, com necessidade de manobras de proteção. Três pacientes (9,7\%) apresentaram nível ruim de disfagia, necessitando de via alternativa de alimentação.

No grupo estudado, não houve diferença significativa quando comparados o grau de penetração/aspiração e a severidade da disfagia com o estadiamento, o local do tumor, recidivas ou óbito.

Todos os pacientes que apresentaram recidivas locais não tinham falecido até a data do último seguimento (agosto/03), e os que faleceram não apresentaram recidivas. Isto é, os que apresentaram recidivas podem ter tido um tempo maior para esta ocorrência, que pode ser atribuído ao estado geral do paciente.

\section{Conclusão}

Este estudo descreve a função de deglutição de pacientes submetidos ao protocolo de preservação de laringe e hipofaringe a curto prazo (média de quatro meses após o término do tratamento). Novos estudos são necessários para analisar a evolução desses pacientes após um período maior. Além disso, estas análises foram realizadas baseando-se em avaliações do ponto de vista do fonoaudiólogo. Sugerimos avaliações dentro da perspectiva do paciente para a compreensão do quanto as alterações analisadas interferem na qualidade de vida dos mesmos.

Conflito de interesse: não há.

\section{SUMMARY \\ SWALlowing AFter CHEMOTHERAPY AND RADIOTHERAPY FOR LARYNGEAL AND HYPOPHARYNGEAL CARCINOMAS}

The main goals of the larynx preservation protocol are eradication of cancer and preservation of a functional larynx with maintenance of respiration, phonation and swallowing. Few studies, however have addressed functional outcomes.

OBJECTIVE. Functional evaluation of oropharyngealswallowing in patients enrolled in alarynx preservation protocol at the Hospital do CâncerAC Camargo.

METHODS. Evaluation of swallowing was performed by videofluoroscopy in 3 I patients, focusing on: oropharyngeal motility disorders, stasis, laryngeal penetration, aspiration and severity of dysphagia.

RESULTS. Swallowing analysis: 5 patients showed inefficient bolus preparation, 14 had changes in the bolus propulsion; 23 patients had a reduced laryngeal elevation, 26 presented with stasis in the valecula and 14 with stasis in hypopharynx. Nine patients presented silent aspiration. We detected functional swallowing in I I patients; mild dysphagia in 7; mild/moderate in 7; moderate in 3 and severe dysphagia in 3.

CONCLUSION. larynx preservation results in changes of swallowing, ranging in their majority from discrete to moderate. Some patients, however, developed severe dysphagia, and oral feeding was not possible. [Rev Assoc Med Bras 2005; $51(2): 93-9]$

KEY WORDS: Swallowing physiology. Organ preservation. Head and neck cancer. Chemotherapy. Radiotherapy.

\section{REFERÊNCIAS}

I. Crespo A. Propedêutica da laringe. In: Carrara-de Angelis E, et al. A atuação da fonoaudiologia no câncer de cabeça e pescoço. São Paulo: Lovise; 2000. p.53-7.

2. Kowalski LP, Miguel REV, Ulbrich FS. Câncer de laringe. In: Carrara-de Angelis E, Furia CLB, Mourão LF, Kowalski LP. A atuação da fonoaudiologia no câncer de cabeça e pescoço. São Paulo: Lovise; 2000. p.97- 104.

3. Hirsch SM, Caldarelli DD, Hutchinson JC, et al. Concomitant chemotherapy and split-course radiation for cure and preservation of speech and swallowing in head and neck cancer. Laryngoscope. 1991; | 0 1:583-6.

4. Robbins KT, Fontanesi J, Wong FSH, Vicario D, Seagren S, Kumar P. A novel organ 
preservation protocol for advanced carcinoma of the larynx and pharynxn Arch Otolaringol Head Neck Surg 1996; I 22:853-7.

5. Kraus DH, Pfister DG, Harrison LB, Shan JP, Spiro RH, Armistrong JG. Larynx preservation with combined chemotherapy and radiation therapy in advanced hypopharynx cancer. Am Acad Otolaryngol Head Neck Surg 1994;3 I -7.

6. Kotz T, Abrahan S, Bettler JJ, Wadler S, Smith RV. Pharyngeal transport dysfunction consequent to an organ-sparing protocol. Arch Otolaryngol Head Neck Surg 1999; I 25:4 I 0-3.

7. Carrara-de Angelis E, Mourão LF, Furia CLB, Avaliação e tratamento das disfagias após tratamento de câncer de cabeça e pescoço. In: Carrara-de Angelis E, Furia CLB, Mourão LF, Kowalski LP. A atuação da fonoaudiologia no câncer de cabeça e pescoço. São Paulo: Lovise; 2000. p. I 55-62.

8. Werner-Kukuk E, Leden HV, Yanagihara N. The effects of radiation therapy on laryngeal function. J Laryngol Otol 1968;82: I - I 5.

9. Lazarus CL, Logemann JA, Pauloski BR, Colangelo LA, Kahrilas PJ, Mittal BB, et al. Swallowing disorders in head and neck cancer patients treated with radiotherapy and adjuvant chemotherapy. Laryngoscope 1996; 106: I | 57-66.

10. Murry T, Madasu R, Martin A, Robbins KT. Acute and chronic changes in swallowing and quality of life following intra arterial chemoradiation for organ preservation in patients with advanced head and neck cancer. Head Neck 1998;20:3 1-7.

I I. Hilman RE, Walsh MJ, Fischer SG, Wolf GT, Hong WK. Functional outcomes following treatment for advanced laryngeal cancer. Part I: voice preservation in advanced laryngeal cancer. Part II: Laryngectomy rehabilitation: the state of the art in the VA system. Ann Otol Rhinol Laryngol I 998; I 72: I-27.

12. Newman LA, Vieira F, Schwiezer V, Samant S, Murry T, Woodson G, et al. Eating and weight changes following chemoradiation therapy for advanced head and neck cancer. Arch Otololaryngol Head Neck Surg 1998; 124:589-92.

13. Smith RV, Kotz T, Beitler JJ, Walders S. Longterm swallowing problems after organ preservation therapy with concomitant radiation therapy and intravenous hydroxyurea. In Arch Otolaryngol Head Neck Surg 2000; I 26:384-9.

14. Newman LA, Robbins KT, Logemann JÁ, lazarus CL, Hamnera A, Tusant $S$, et al. Swallowing ability after treatment for head and neck cancer with target intra-arterial versus intravenous chemoradiation. Dysphagia 2002; 17: / 84

15. Ansfield FD, Ramirez G, Davis HL, Korbitz BC, Vermund H, Gollin FF. Treatment of advanced cancer of the head and neck. Cancer
1970; 15:78-82.

16. Shrinian MH, Weber RS, Lippman SM, Dimery W, Early CL, Garden AS. Laryngeal preservation by induction chemotherapy plus radiotherapy in locally advanced head and neck cancer: the M.D. Anderson cancer center experience. Head Neck 1994; I 6:39-43.

17. List MA, Siston A, Haraf D, Schumm P, Kies M, Stenson K, Volkes EE. Quality of life and performance in advanced head and neck cancer patients on concomitant chemoradiotherapy: a prospective examination. J Clin Oncol 1999; 17: 1 020-8.

18. Lazarus CL, Logemann JÁ, Kahrilas PJ, Mittal BB. Swallowing recovery in oral cancer patient following surgery, radiotherapy, and hyperthermia. Head Neck 1994; 1 6:259-65.

19. Novaes PERS. Radioterapia em neoplasias de câncer de cabeça e pescoço. In: Carrara-de Angelis $\mathrm{E}$, et al. A atuação da fonoaudiologia no câncer de cabeça e pescoço. São Paulo: Lovise; 2000. p.33-4l.

20. Carrara-de Angelis E, Feher O, Barros APB, Nishimoto IN, Kowalski LP. Voice and swallowing in patients enroled in a larynx preservation trial. Arch Otolaryngol Head Neck Surg 2003; 129:733-8.

Artigo recebido: 12/01/04

Aceito para publicação: 18/10/04

\section{www.ramb.org.br}

\section{A Ramb disponibiliza agora um novo serviço para os nossos colaboradores:}

0 envio de artigos pela internet. Através do nosso publicador, que agiliza o fluxo

de submissão com mais rapidez e versatilidade, você poderá enviar seu artigo para publicação na Ramb. Experimente: é muito mais cômodo, muito mais rápido e você ainda poderá acompanhar on line o curso de sua apreciação.

\section{Acesse www.ramb.org.br}

